\title{
DNA Methyltransferase
}

National Cancer Institute

\section{Source}

National Cancer Institute. DNA Methyltransferase. NCI Thesaurus. Code C16508.

DNA Methyltransferases are a subclass of transferase enzymes that catalyze the transfer of a methyl group from a donor compound to DNA. 